\title{
Technique for the electric and magnetic parameter measurement of powdered materials
}

\author{
R. Kubacki, L. Nowosielski \& R. Przesmycki \\ Faculty of Electronics, Military University of Technology, Poland
}

\begin{abstract}
A measurement technique for the electric and magnetic properties of powdered ferrites has been developed. This technique allows one to determine relative permittivity and permeability of powdered materials. Measurements were taken in a coaxial transmission line, which guarantees the broadband frequency measurements. The calculations were based on evaluation of the scattering parameters of measured powdered material and two plastic walls. The values of relative permittivity $\left(\varepsilon^{\prime}, \varepsilon^{\prime \prime}\right)$ and permeability $\left(\mu^{\prime}, \mu^{\prime \prime}\right)$ have been done for ferrite powder in the frequency range from $200 \mathrm{MHz}$ to $1200 \mathrm{MHz}$.
\end{abstract}

Keywords: absorbing materials, permittivity and permeability measurements.

\section{Introduction}

With the increasing quantity of devices emitting electromagnetic radiation, there is a need to develop and introduce into practice materials and techniques to protect against unwanted radiation. This is important for EMC purposes as well as for the protection of people against the harmful radiation. There are two ways in which materials can shield against radiation: by reflecting or by absorbing the incident electromagnetic energy. The ideal absorber should have low reflectivity and a high value of absorption of the incident electromagnetic energy. The electromagnetic field incident to the boundary surface of any material is reflected and the level of reflected energy is a function of the internal parameters of the material. In general, the shielding effectiveness of a material or configuration of materials is a measure of its ability to attenuate electromagnetic energy. This ability depends on both its reflection and absorption properties. Energy not being reflected or absorbed by the material is transmitted from one side to the other. 
For a given material, the amount of energy transmitted has a complex dependence upon the angle of incidence and polarization of the incoming electromagnetic wave.

There are a few ways to obtain the minimal value of reflected power; however, the most often applied technique is to cover the protected walls with graphite materials having a pyramidal layer structure. Unfortunately, such structures operate correctly at rather higher (microwave) frequencies.

At frequencies lower than $10 \mathrm{MHz}$, such a technique with a pyramidal layer structure does not guarantee the decrease of the level of reflected energy because, generally speaking, the wavelength is much bigger than the highest of the pyramids. Another way to obtain low reflectivity of material is to use materials having not only electric properties, but also magnetic properties. Materials with such properties are ferrite materials. Nowadays, walls in anechoic chambers are covered by broadband absorbers composed of two layers. The first one is pyramidal structure polyurethane foam loaded with graphite and the second layer is a sintered ferrite. Commercially available monolithic ferrite materials guarantee good absorption properties and a low level of reflected field; however, in many practical uses such a solid form of absorber cannot be introduced. From this point of view there is a need to develop absorbers that can fulfill the following requirements:

- operate at desirable frequencies or over a wide frequency band;

- be a solid or flexible form of material;

- have the desirable level of reflectivity and absorption.

New materials can be used for specific studies, e.g. stealthness, but in this case rather composite materials should be developed.

It is possible to simulate the properties of an absorber having the above properties; however the constitutive material parameters, e.g. relative permittivity $\varepsilon_{r}$ and permeability $\mu_{r}$, of such materials or any component of these materials should be determined. In many cases components are in powder form. In fact, most of absorbing material is usually composed of powder components joined together with an epoxy bonder.

The important thing is to assess the electric and magnetic parameters of the components of the mixture, being in powder form before their mixing.

\section{Network analyzer measurements}

There are many methods that can be used to determine the permittivity $\varepsilon_{r}$ and the permeability $\mu_{r}$ of materials. Resonant cavity techniques offer high accuracy of measurements but they can only be used mainly for one frequency. On the other hand, waveguide methods make possible measurements over a wide frequency band but they have some disadvantages when measured materials are lossy. Nevertheless, measurements in waveguides are based on measuring of the scattering parameters $\left(S_{i k}\right)$.

In this paper measurements of scattering parameters were taken according to the approach given in Baker-Jarvis [1]. This approach refers to toroidal material 


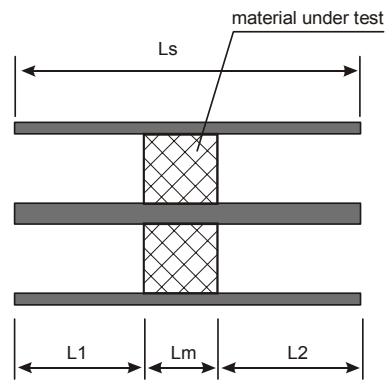

Figure 1: Coaxial transmission line with a sample of material under test.

under test inserted into a transmission coaxial line - fig. 1. In this method the specimen is treated as homogeneous.

Baker-Jarvis [1] described the technique of determining values of the propagation constant $\left(\gamma_{m}\right)$ and characteristic impedance $\left(Z_{m}\right)$, taking into account the measured scattering parameters. However, in order to determine the values of $\gamma_{m}$ and $Z_{m}$ of the material under test $(\mathrm{Lm})$, the scattering parameters of this sample should be extracted from the scattering parameters of whole coaxial section $(L s)$. In this case airline sections having lengths $L 1$ and $L 2$ must be compensated. This is an important task to minimize measurement errors due to uncontrolled displacement of the sample. To minimize these errors a method was developed taking into account the nonlinear optimization of the calculation with inserted airlines sections [1].

Measurements of the scattering parameters of the sample inserted into a coaxial line were taken using a vector network analyzer system. However, special additional accessories are necessary for the calibration process. Effective auxiliary tools for calibration were developed and described in [2, 3]. These tools are in fact three airlines with different lengths depending on the frequency range measurements. This special calibration process guarantees the highest accuracy of measurements. Taking into account calibration evaluation, the computational program can compensate for errors caused by any potential displacement of the sample.

The above-mentioned measurement technique is also called the reflection/transmission method. This method is well adapted to broadband characterization of isotropic and homogeneous materials; however, there are some disadvantages to this technique. On one hand, at frequencies where $L_{m} \sqrt{\varepsilon_{r} \mu_{r}}=n \lambda / 2$ (n - integer) $S_{11}$ vanishes and in such case noise affects measurements. Consequently, the network analyzer phase uncertainty is large. On the other hand, when material is lossy (or thickness of a sample is large) the value of $S_{21}$ can also vanish. So, the reflection/transmission method should be applied to the characterization of several materials, where permittivity and permeability are approximately known. 


\section{Measurement method of the relative permittivity and permeability of powdered materials}

There are few waveguide methods that allow one to measure the complex relative permittivity $\varepsilon_{r}$ of non-solid materials. In the case of liquid materials, measurement methods are easy to realize, especially when they are lossy. For example, a typical waveguide setup used for measuring liquid biological tissues consists of a coaxial open-ended line applied to a liquid surface (Kubacki et al. [4]). In this case the measurement of the complex reflection coefficient is sufficient to determine the values of two parameters $\varepsilon_{r}\left(\varepsilon^{\prime}, \varepsilon^{\prime \prime}\right)$ of the liquid. However, when the material also has magnetic properties $\mu_{r}\left(\mu^{\prime}, \mu^{\prime}\right)$, such measurements based on determining only the reflection coefficient are not sufficient. In this case the transmission coefficient should also be measured. This could be done using waveguides or coaxial lines where values of two complex scattering parameters can be measured.

The method described in this paper allows one to measure complex relative permittivity $\varepsilon_{r}$ and permeability $\mu_{r}$ of non solid (mainly powder) materials over a wide frequency range - from a few $\mathrm{kHz}$ to a few $\mathrm{GHz}$. The technique of measurement has been based on a method developed for coaxial transmission lines where the material under test must have toroidal form. In the proposed method, the material under test is inserted into a coaxial line between two plastic walls as is shown in fig. 2. This allows one to measure powdered materials. The values of electric $\left(\varepsilon^{\prime}, \varepsilon^{\prime \prime}\right)$ and magnetic parameters $\left(\mu^{\prime}, \mu\right.$ ') of measured powder are extracted in a mathematical evaluation from the measured data of scattering parameters of the whole sample $\left(S_{11}, S_{22}, S_{12}, S_{21}\right)$.

Mathematical evaluation that allows one to extract the electromagnetic data of the measured powder from measured data of the whole sample has been derived on the basis of scattering parameters describing two-port networks as is presented in fig.3. In this figure, the scattering parameters of plastic walls were defined as $E_{i k}$ while the scattering parameters of measured powdered material are defined as $S_{i k}$.

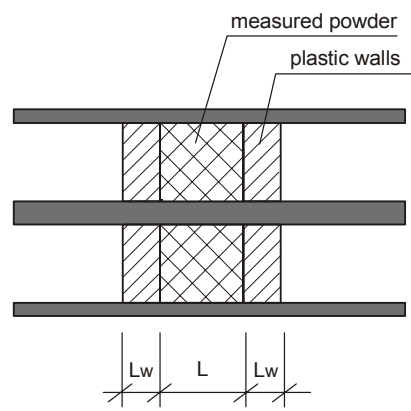

Figure 2: The sketch of the coaxial line with a sample of material under test between two plastic walls. 


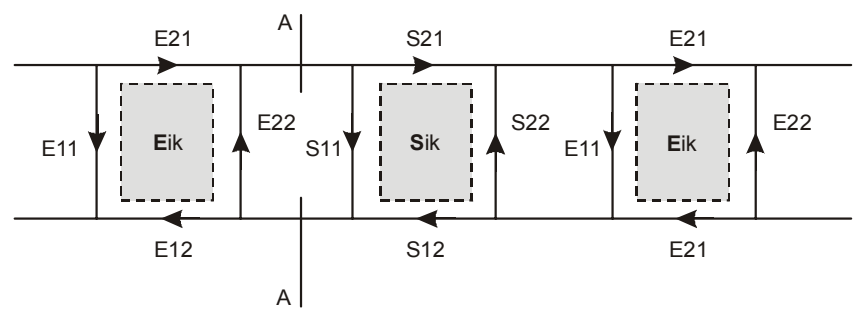

Figure 3: $\quad$ Scattering parameters of plastic walls $\left(E_{i k}\right)$ and powder under test $\left(S_{i k}\right)$.

Taking into account the reciprocal and source-free networks, the scattering matrix satisfies the following condition: $S_{i k}=S_{k i}$. In our case for symmetrical twoport networks: $S_{11}=S_{22}, S_{12}=S_{21}$ and also $E_{11}=E_{22}, E_{12}=E_{21}$. For each network it is possible to formulate the following scattering equations:

$$
\left[\begin{array}{l}
b_{1} \\
b_{2}
\end{array}\right]=\left[\begin{array}{ll}
S_{11} & S_{12} \\
S_{21} & S_{22}
\end{array}\right]\left[\begin{array}{l}
a_{1} \\
a_{2}
\end{array}\right]
$$

Scattering matrix (1) refers to a single network and for the sample composed of three layers such matrix becomes much more complicated. To receive mathematical formulas that allow one to extract scattering parameters of networks representing the powder substrate, the so called graph method of calculation has been introduced. The graph method has been derived from the theory of power flow in the network branches. As an example of how to use graph method in practice the reflection coefficient at the gate A-A (fig. 3) has been presented in fig. 4 .

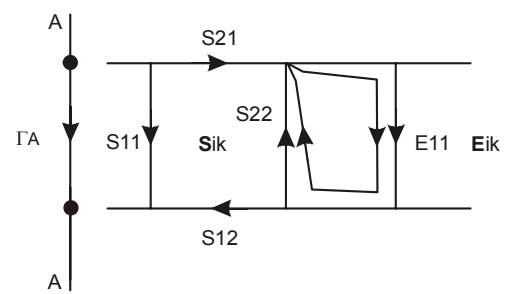

Figure 4: The graph method used for the example analysis of the reflection coefficient at the gate A-A.

The final formulas of the scattering parameters $S w_{i k}$ of the whole sample (material under test and two plastic walls) are as follows:

$$
S w_{11}=E_{11}+E_{21} \frac{\left(1-E_{11} S_{11}\right) S_{11}+E_{11} S_{21}^{2}}{\left(1-E_{11} S_{11}\right)^{2}-E_{11}^{2} S_{21}^{2}}
$$




$$
S w_{21}=\frac{E_{21}^{2} S_{21}}{\left(1-E_{11} S_{11}\right)^{2}-E_{11} S_{21}}
$$

where $S w_{i k}$ represents the scattering parameters of whole sample.

Formulas (2) and (3) allow one to determine the scattering parameters of the specimen $\left(S_{11}, S_{2 l}\right)$ from the scattering data of whole sample $\left(S w_{11}, S w_{21}\right)$.

\section{Experiments and results}

Measurements of complex relative permittivity and permeability were carried out using a vector network analyzer (VNA). This system consisted of a $7 \mathrm{~mm}$ coaxial airline, equipped with measurement cables and LPC7 connectors. The center conductor of the coaxial airline is $3,04 \mathrm{~mm}$ in diameter to receive the $50 \Omega$ characteristic impedance of the holder. The system measures the magnitude and phase of four scattering parameters $\left(S_{11}, S_{22}, S_{12}, S_{21}\right)$. First of all the empty coaxial airlines were employed to calibrate the system. The additional tools consisted of three auxiliary airlines that were used to realize the calibration. These airlines are sample holders as well as calibration tools. They have been presented in fig. 5. The whole measurement setup is presented in fig. 6 .

Taking into account that various components of the VNA introduce magnitude and phase uncertainties, the calibration process is important in order to obtain the highest accuracy of measurements [1]. Calibration removes the systematic measurement uncertainties of the system. The calibration coefficients were determined by solving a set of simultaneous equations generated from the linear fractional transformation. After calibration, when the system was operated with the errors correction, the measurements were updated by the calibration coefficients. Data of calibration process and measurements of the sample were acquired using the MultiCal program, than they were processed with a special program developed for extracting values of permittivity and permeability.

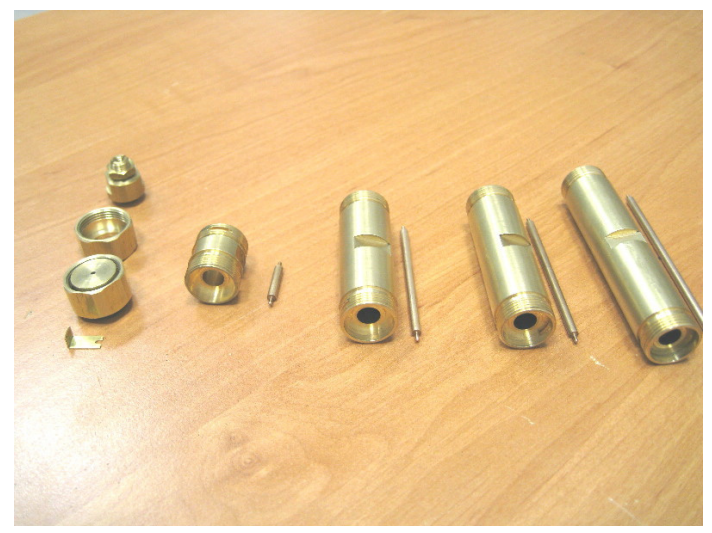

Figure 5: Coaxial airlines as sample holders and/or calibration tools. 


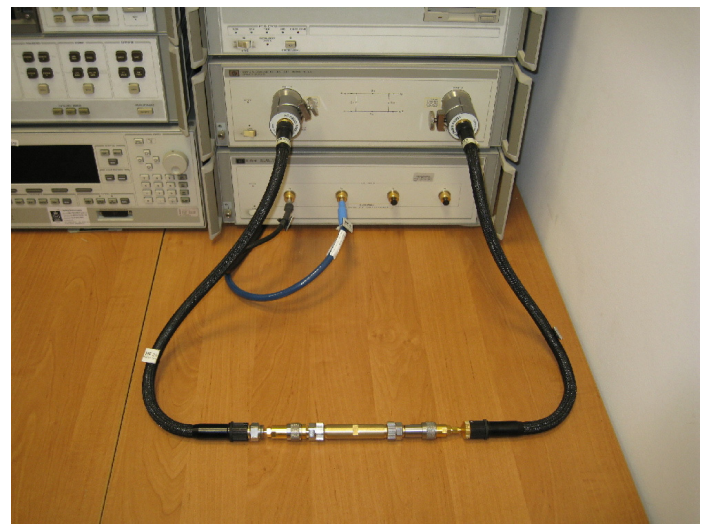

Figure 6: S-parameter test of the vector network analyzer and a coaxial line with sample.

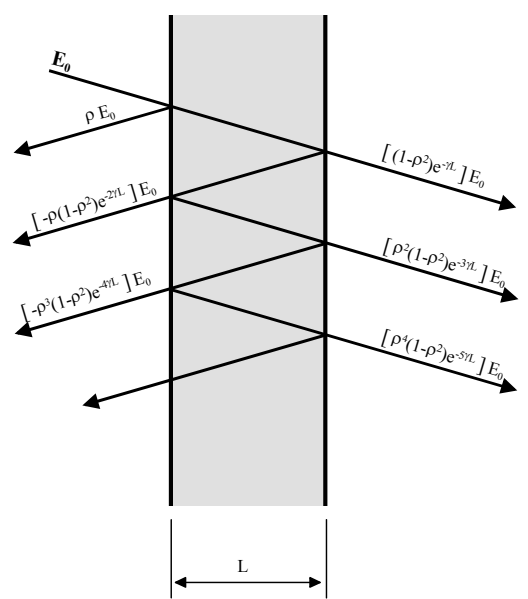

Figure 7: The infinite reflections and transmissions according to the theory of optical rays.

The final formulas of the scattering parameters of a powder $\left(S_{11}, S_{21}\right)$ were extracted from the scattering parameters of the whole sample (2) and (3). On the other hand, these scattering parameters could be determined by analyzing the electric field at the specimen interface. In order to determine the material properties from scattering data there is a need to introduce the relationship of the electric field incident at the boundary of the specimen and propagate through the materials. The reflection and transmission coefficients can be interpreted as the infinite summation of optical rays when the incident electromagnetic field is treated as a plane wave. Such a situation is typical in a coaxial line where the TEM mode is propagated. The infinite reflections and transmissions are shown in fig. 7. 
The reflection and transmission of rays have been presented according to the theory of optics (fig. 7), taking into account two boundary conditions: "air/sample" as well as "sample/air". The reflection coefficient $\left(S_{11}\right)$ consists of infinite rays in which the first one is reflected at the first interface air/sample $(\rho)$ and all the others are transmitted, arising from multiple reflections inside the sample. On the other hand, the transmission coefficient $\left(S_{21}\right)$ is the infinite summation of the rays transmitted to the second interface "sample/air". This infinite sum of components is in fact a geometric series yielding to the following final sums:

$$
\begin{aligned}
& S_{11}=\rho \frac{1-e^{-2 \gamma L}}{1-\rho^{2} e^{-2 \gamma L}} \\
& S_{21}=\frac{\left(1-\rho^{2}\right) e^{-\gamma L}}{1-\rho^{2} e^{-2 \gamma L}}
\end{aligned}
$$

where, in the coaxial transmission line with the fundamental mode TEM:

$$
\begin{array}{ll}
\rho \text { - reflection coefficient: } & \rho=\frac{\sqrt{\mu_{r}}-\sqrt{\varepsilon_{r}}}{\sqrt{\mu_{r}}+\sqrt{\varepsilon_{r}}} \\
\gamma \text {-propagation constant: } & \gamma=j \omega \sqrt{\varepsilon_{r} \mu_{r}}
\end{array}
$$

Formulas (4) and (5) are functions of unknown parameters of $\varepsilon_{r}$ and $\mu_{r}$ of the specimen. Values of these parameters $\left(\varepsilon^{\prime}, \varepsilon^{\prime}, \mu^{\prime} \mu^{\prime \prime}\right)$ can be obtained by solving the above complex equations (4) and (5). In this case the iterative procedure yields to a very stable solution for a specimen of arbitrary length.

For the purpose of the validation of the solution of the sample of solid material, the measurement in the configuration with two plastic walls was used. Then, the values of $\varepsilon^{\prime}, \varepsilon^{\prime}, \mu^{\prime} \mu^{\prime \prime}$ were determined using the proposed method. The obtained data were confirmed by measuring this solid sample (without plastic walls) using the reflection/transmission method described in $[1,3]$. The measured values of $\varepsilon^{\prime}, \varepsilon^{\prime \prime}, \mu^{\prime}$ and $\mu^{\prime \prime}$ by the proposed method were within good accuracy with the reflection/transmission method.

As an example of powdered material measurements, the values of complex permittivity and permeability of ferrite powder have been measured using the proposed method. Ferrite powder was received in a ball mill from the solid state material (spinel class ferrite, named G-175) to receive granulate $(0,2-2) \mu \mathrm{m}$. For this powdered material, relative values of permittivity $\left(\varepsilon^{\prime}, \varepsilon^{\prime \prime}\right)$ versus frequency were presented in fig. 8 , while relative values of permeability $\left(\mu\right.$ ', $\left.\mu^{\prime \prime}\right)$ were presented in fig. 9 .

The measured values of permittivity $\left(\varepsilon^{\prime}, \varepsilon^{\prime \prime}\right)$ and permeability $\left(\mu^{\prime}, \mu^{\prime \prime}\right)$ of the ferrite powder versus frequency were presented in figs. 8 and 9 . However, the obtained data for the powdered ferrite were significantly lower than that of solid state ferrite. This is because the magnetic domains of ferrite were destroyed during the milling process. Further investigation should be undertaken to characterize the relationship between the volume of granulates and the decreasing permeability. 


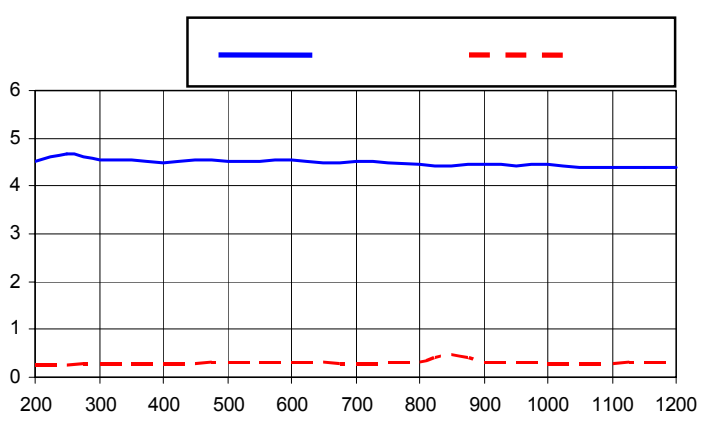

Figure 8: Values of the relative permittivity of ferromagnetic powder in the function of frequency.

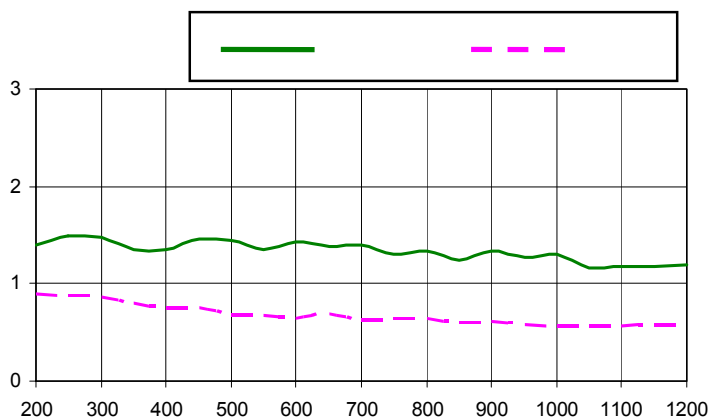

Figure 9: Values of the relative permeability of ferromagnetic powder in the function of frequency.

In the described measurements all grains of powder were relatively small, compared to the wavelength, to receive homogeneous material. Measurement problems can appear in the measurement transmission line when material under test is heterogeneous. This can happen when materials contain inclusions or when grains of powder are not small compared to the wavelength. In these conditions the higher order modes can be excited in the sample by diffraction on the heterogenities. So, the parameters $\varepsilon_{\text {eff }}$ and $\mu_{\text {eff }}$ of such a sample cannot be deduced in classical calculation because it supposes that only the dominant mode propagates. Moreover, in such structures with local inclusions, the local resonances are excited. This causes a lack of energy to be measured when higher order modes appear. This is due to metallic losses in the coaxial line and inclusions. 


\section{Conclusions}

The measurement technique of electric and magnetic properties of powdered ferrites has been developed. This technique seems to be useful for lossy materials. Based on this technique, values of relative permittivity $\left(\varepsilon^{\prime}, \varepsilon^{\prime \prime}\right)$ and permeability $(\mu$ ', $\mu$ ') of ferromagnetic powders can be measured in the frequency range from $200 \mathrm{MHz}$ to $1200 \mathrm{MHz}$. The aim of this work was to investigate the measurement technique of powdered materials, because it could be an important tool for simulating the final properties of absorbing materials composed of conductor and ferrite materials. However, it turned out that in the case of ferrite powder such an attempt falls down because the values of relative permeability $\left(\mu^{\prime}, \mu^{\prime \prime}\right)$ of powder was significantly lower than that of solid state ferrite. Further investigation should be directed to find the difference in permeability between the powdered and solid state ferrites.

\section{Acknowledgement}

The authors would like to thank Ryszard Frender from the Telecommunications Research Institute, Warsaw, Poland for providing ferrite powders for the measurements.

\section{References}

[1] Baker-Jarvis J., Transmission/reflection and short-circuit line permittivity measurements, NIST Technical Note, 2005.

[2] Marks R.B., A multiline method of network analyzer calibration, IEEE Trans. Microwave Theory Tech., 42, pp. 1205-1215, 1991.

[3] Wiatr W., Frender R., Żebrowski M., „Characterization of microwave absorbing materials using a wideband T/R measurement technique", Conference Proceedings on International Conference on Microwaves, Radar and Wireless Communications, Wrocław, Poland, 2, pp. 475-478, 2008.

[4] Kubacki R., Sobiech J., Wardak K., The comparison of dielectric properties of young and mature animal tissues in microwaves, Przeglad Elektrotechniczny, 5, pp. 43-45, 2006. 